\title{
Numerical methods for the travelling wave solutions in reaction-diffusion equations
}

\author{
V. Gubernov G. N. Mercer H. S. Sidhu \\ R. O. Weber*
}

(Received 1 June 2001)

\begin{abstract}
In this work we consider how shooting and relaxation methods can be used to investigate propagating waves solutions of PDEs. Particular attention is paid to overcoming some of the numerical difficulties. The linear stability of these solutions are analyzed by using the Evans function approach. As an illustration, we shall apply the above methods to an autocatalytic reaction involving two diffusing chemicals in one spatial dimension. Prospects of further work are also discussed.
\end{abstract}

* School of Mathematics and Statistics, University of New South Wales at Australian Defence Force Academy, Canberra, ACT 2600, Australia. mailto:vlad@ma.adfa.edu.au, mailto:g.mercer@ma.adfa.edu.au, mailto:h.sidhud@ma.adfa.edu.au, mailto:r.weber@ma.adfa.edu.au, respectively.

${ }^{0}$ See http://anziamj.austms.org.au/V44/CTAC2001/Gube for this article, (C) Austral. Mathematical Soc. 2003. Published 1 April 2003. ISSN 1446-8735 


\section{Contents}

1 Introduction

C272

2 Statement of problem

C273

3 Travelling front solution

C275

4 Stability analysis

C277

5 Compound matrix method

C283

6 Conclusions

C285

References

C287

\section{Introduction}

Many physical, chemical and biological phenomena such as combustion waves, optical solitons, chemical reactions, propagation of dominant genes and nerve pulses etc. are modelled by nonlinear Partial Differential Equations (PDEs) exhibiting travelling wave solutions $[1,2,3,4,5]$.

Steady propagating solutions in the form of a pulse or front travelling without change of form and speed are of special interest. In this case the governing PDEs can be reduced to a system of Ordinary Differential Equations(ODEs) by introducing a moving coordinate frame. The form of the front(pulse) is then given by the solution of the system of ODEs, which usually can be found only numerically as a solution of a two-point boundary value problem (BVP).

This BVP can be stated in the following form: we want to find 
the solution of $N$ coupled first-order ODEs, satisfying $n_{1}$ boundary conditions at the starting point $x_{1}$, and $n_{2}=N-n_{1}$ boundary conditions at the final point $x_{2}$. Once the problem is formulated in the form of a two-point BVP, methods for numerical integration such as shooting and relaxation [6] can be employed to find the form of the steady propagating front(pulse). In section 3 we illustrate the application of these methods to the example of an autocatalytic reaction involving two chemical species in one spatial dimension. Following the work in [7] we obtain major properties of travelling front solution such as the dependence of the speed of the propagating front on the parameters of the system.

Steady propagating solutions can lose stability as we change parameters of the system. For example, in $[7,8]$ it was shown that the travelling fronts lost their stability in an oscillatory manner and appears to exhibit a period doubling route to chaos. To investigate the travelling front stability, the corresponding linear stability problem was solved using the Nyquist technique via an Evans function [9]. In section 4 we introduce the definition of the Evans function and describe its relation to the linear stability problem.

In this case, however, the conventional algorithm for a calculating the Evans function introduced in [9] encounters serious obstacles due to the necessity of numerical tracing of two solutions with different rates of exponential growth or decay. In section 5 we show how this difficulty was overcome by using the compound matrix method [11, 12, 13, 14, 15, 16].

\section{Statement of problem}

Let us consider an autocatalytic reaction involving two chemicals (the autocatalyst $u(x, t)$, and the reactant $v(x, t))$ in one spatial 
dimension. In non-dimensional coordinates, the equations governing this process can be found in [7] and are

$$
u_{t}=u_{x x}+v f(u), \quad v_{t}=\tau v_{x x}-v f(u),
$$

where $\tau$ is the inverse of the Lewis number (the ratio of the diffusion rates of the two chemicals) and the reactant rate is

$$
f(u)= \begin{cases}u^{m}, & u \geq 0 \\ 0, & u<0\end{cases}
$$

where $m$ is the reaction order. We assume the following boundary conditions:

$$
\begin{aligned}
& u(x, t) \rightarrow 1, v(x, t) \rightarrow 0 \text { as } x \rightarrow-\infty \\
& u(x, t) \rightarrow 0, v(x, t) \rightarrow 1 \text { as } x \rightarrow+\infty
\end{aligned}
$$

Solution of (1) with (3) are sought by assuming the form $u(x, t)=$ $U(x-c t)$ and $v(x, t)=V(x-c t)$ of a front travelling with speed $c$. After substitution of this solution into (1) it is easy to obtain

$$
U_{\xi \xi}+U^{m} V=-c U_{\xi}, \quad \tau V_{\xi \xi}-U^{m} V=-c V_{\xi},
$$

and the boundary conditions

$$
\begin{aligned}
& U(\xi) \rightarrow 1, V(\xi) \rightarrow 0 \text { as } \xi \rightarrow-\infty \\
& U(\xi) \rightarrow 0, V(\xi) \rightarrow 1 \text { as } \xi \rightarrow+\infty
\end{aligned}
$$

where $\xi=x-c t$ is a coordinate in the moving frame. It can be shown (see [7] for details) that (4) has a unique solution if and only if $c$ is greater than a minimum value $c_{\star}(m, \tau)$. All fronts travelling with speed $c>c_{\star}$ decay algebraically as $\xi \rightarrow+\infty$. On the contrary, the front with $c=c_{\star}$ decays exponentially as $\xi \rightarrow+\infty$. In the discussion that follows, we will only be interested in the latter case. System (4) has two fixed points: $S_{1}$ of $U=1, V=0$; and $S_{2}$ of $U=0$, $V=1$. Equations obtained through linearization of (4) near $S_{1}$ 
have only one exponentially unstable solution $(U-1, V) \sim \mathbf{q} e^{h \xi}$, where $\mathbf{q}=(-1, h(h+c))$ and $h=\left(-c+\sqrt{c^{2}+4 \tau}\right) / 2 \tau$. Any solution $U(\xi), V(\xi)$ of (4) tending to $S_{1}$ as $\xi \rightarrow-\infty$ approaches $S_{1}$ as $\mathbf{q} e^{h\left(\xi-\xi_{0}\right)}$, where $\xi_{0}$ depends on initial conditions. Similarly, linear analysis of (4) near $S_{2}$ shows that any solution of (4) satisfying the right boundary conditions can be approximated for $\xi \rightarrow+\infty$ as $(U, V-1)=\left(k_{1} e^{-c\left(\xi-\xi_{0}\right)}, k_{2} e^{-c\left(\xi-\xi_{0}\right) / \tau}\right)$ where $k_{1}$ and $k_{2}$ are constants. The travelling front solution of (1) corresponds to the solution of (4) connecting two fixed points and satisfying the given asymptotic behavior.

\section{$3 \quad$ Travelling front solution}

It is convenient to rewrite (4) as the system of first-order ODEs

$$
\dot{y}_{i}=f_{i}(\mathbf{y}, \xi),
$$

where $i=1, \ldots, 4, y_{1}=U, y_{2}=U_{\xi}, y_{3}=V, y_{4}=V_{\xi}$, and $f_{1}=y_{2}$, $f_{2}=-f\left(y_{1}\right) y_{3}-c y_{2}, f_{3}=y_{4}, f_{4}=\left(f\left(y_{1}\right) y_{3}-c y_{4}\right) / \tau$. As a first step we will find an approximate solution of (6) using a shooting method [6]. Numerically we integrate (6) from $\xi=-\ell_{1}$ to $\xi=\ell_{2}$, where $\ell_{1}$ and $\ell_{2}$ are considered to be sufficiently large. We used the fourth order Runge-Kutta method of integration. On the left boundary we define initial conditions, which satisfy the asymptotic behavior for $\xi \rightarrow-\infty$ given above, as

$$
\mathbf{y}=\left(1-e^{-h \ell_{1}},-h e^{-h \ell_{1}}, h(h+c) e^{-h \ell_{1}}, h^{2}(h+c) e^{-h \ell_{1}}\right)^{T},
$$

where $T$ stands for the transpose. Note that due to the translational symmetry of (1) we can take the initial conditions at any point lying on the unstable manifold of $S_{1}$. Linear analysis of (6) shows that (7) approximates it near $S_{1}$, where $\ell_{1}$ can be considered as a parameter 
characterizing the distance between $S_{1}$ and point on the unstable manifold. Furthermore, the approximation (7) holds if $h \ell_{1} \gg 1$.

On the right boundary we cannot prescribe the value of any component of the vector $\mathbf{y}$; except to say that it must satisfy definite asymptotic behavior for large $\xi$. For this reason we use the Cauchy boundary conditions

$$
B_{1}=c y_{1}+y_{2}=0, \quad B_{2}=c y_{3}+\tau y_{4}=0,
$$

where $\mathbf{y}$ is taken at $\xi=\ell_{2}$. By assuming that $c$ is the free parameter, we make a guess for the value of $c$ and integrate (6) with initial conditions (7). We consider the discrepancy of one of the conditions on the right boundary ( $B_{1}$, for instance). Then, by using NewtonRaphson method the root of equation $B_{1}(c)=0$ can be found with desired accuracy. We exploit another condition $B_{2}(c)=0$ to check the accuracy of the method.

We can now make use of the solution obtained from the shooting as a guess for a more accurate scheme - the relaxation. In contrast to the shooting method described previously, the relaxation scheme improves the guess solution on every mesh point. The system of $N$ ODEs given by (6) is replaced by finite-difference equations(FDE) on $M$ mesh points. We use a second-order FDE relating values at two points $k$ and $k-1$

$$
\mathbf{y}_{k}-\mathbf{y}_{k-1}=\left(\xi_{k}-\xi_{k-1}\right) \mathbf{f}\left[\left(\mathbf{y}_{k}-\mathbf{y}_{k-1}\right) / 2\right] .
$$

Higher order formulas are discussed in [17]. Now we have a system of equations for $M \times N$ variables, which can be solved using a multidimensional Newton's method (see [6] for details). In our case we have the system of four ODEs (6) with $c$ as a free parameter which also has to be found. This problem is reducible to standard boundary value problem [6]. We introduce new variable $y_{5} \equiv c$ and another differential equation $\dot{y}_{5}=0$. Now we have five equations. We 
take three boundary conditions from (7) on the left boundary and two Cauchy conditions (8) on the right boundary. The relaxation method is then used to calculate the solution of the problem (4-5). Results obtained for $\tau=0.1$ and $m=2,13$ are shown in Figure 1.

As we increase $m$ for fixed $\tau$ the autocatalyst $u$ front becomes flatter and the interval of the integration $\left(-\ell_{1}, \ell_{2}\right)$ has to be increased. In contrast, the front of reactant $v$ steepens and we must decrease the step of integration near the point of the maximal slope. This imposes additional difficulties if we want to investigate properties of the travelling front solution such as speed $c$ as a function of the parameters $\tau$ and $m$. The best way to overcome this obstacle is to introduce the tangential transformation $\xi=\arctan (\theta)$ which maps the infinite interval $\xi \in(-\infty, \infty)$ onto $\theta \in(-\pi / 2, \pi / 2)$. Then we modify the equations (4) and introduce the uniform mesh on $(-\pi / 2, \pi / 2)$. Figure 2 shows the speed of the front solution as a function of parameter $m$ for fixed values of $\tau$. For every point on the parameter plane $(m, \tau)$ under consideration, we use the shooting method as a prediction and then correct the results with the relaxation method outlined above.

Neither the shooting nor the relaxation scheme allows us to analyze the stability of steady propagating solutions. In the next section a linear stability problem of (1) is considered.

\section{$4 \quad$ Stability analysis}

As a first step in the analysis of travelling wave stability we linearize (1) around the front solution

$$
u(\xi, t)=U(\xi-c t)+\varphi(\xi-c t, t), \quad v(\xi, t)=V(\xi-c t)+\chi(\xi-c t, t),
$$




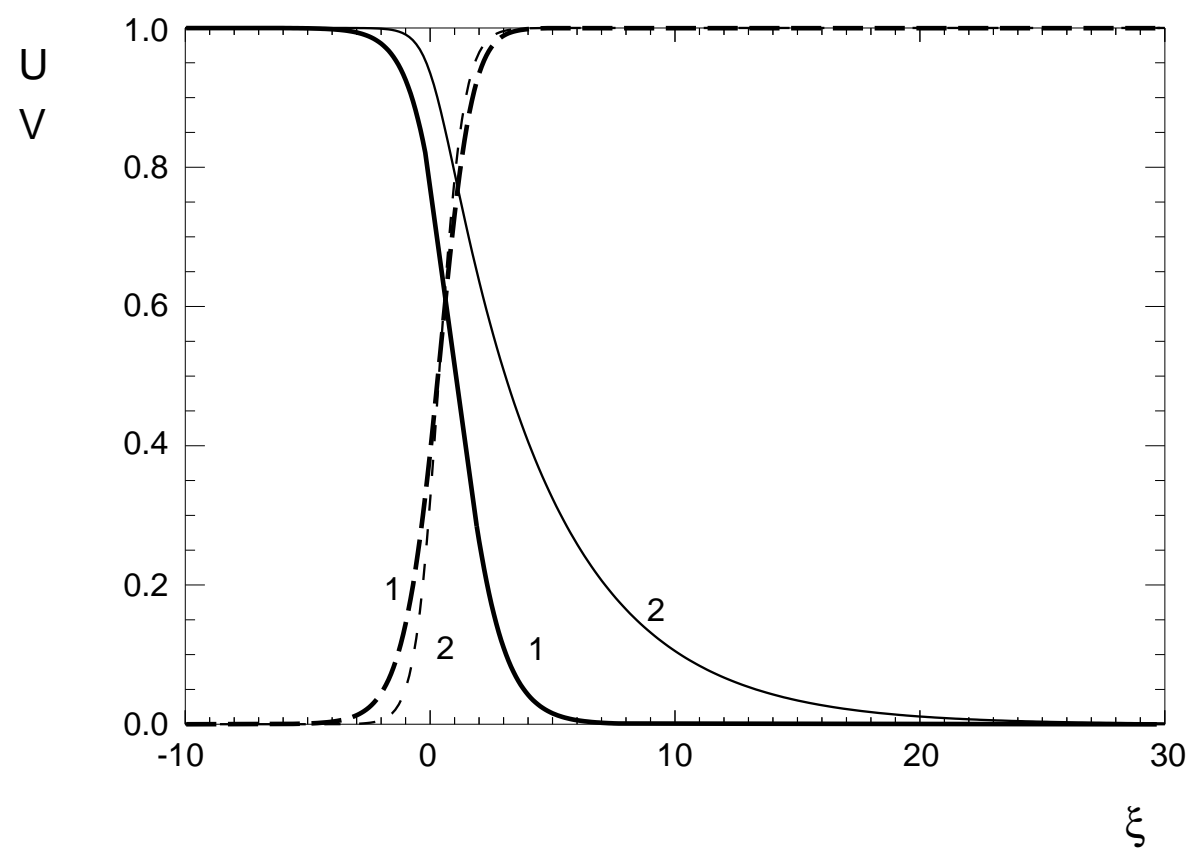

FiguRE 1: Solutions of (4) obtained by relaxation method for parameters $\tau=0.1$ and $m=2$ (curves 1 ), $m=13$ (curves 2). Solid curves correspond to $U(\xi)$ and dashed to $V(\xi)$ 


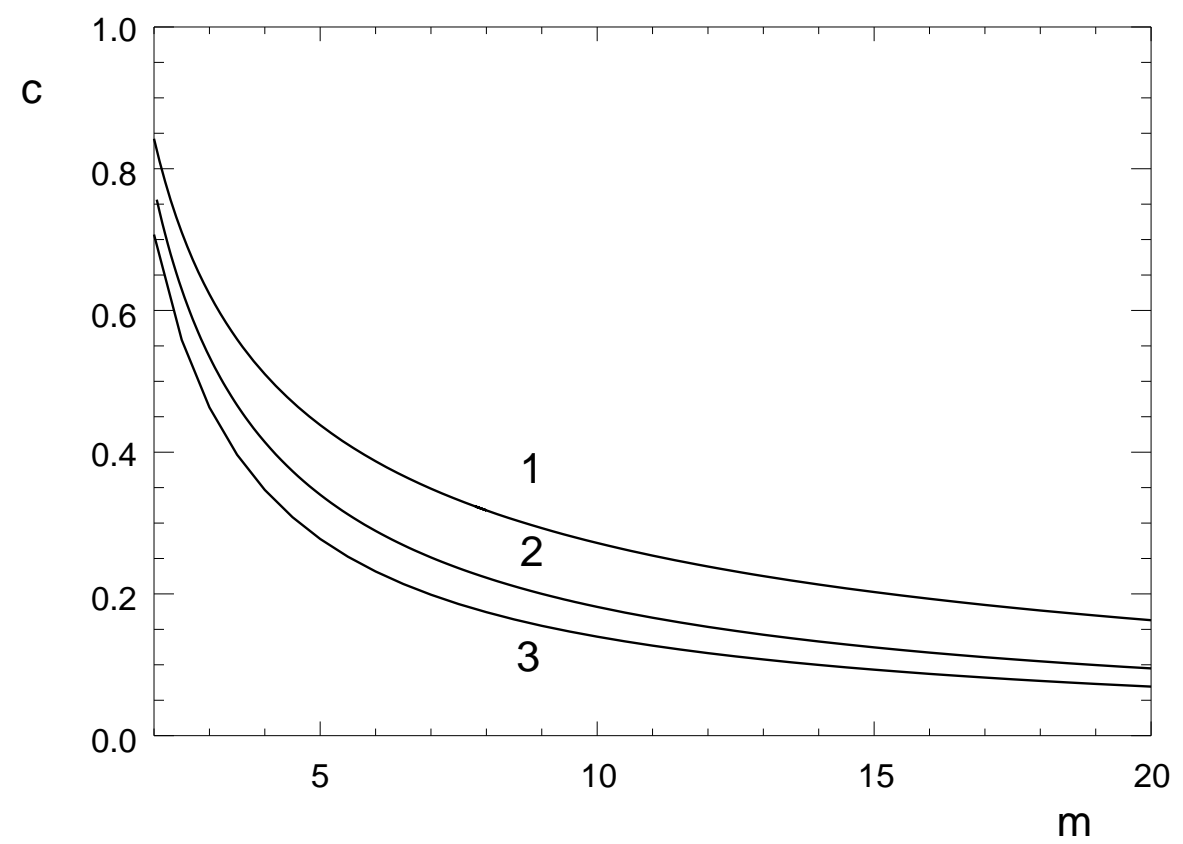

FiguRE 2: Dependence of front speed $c$ on parameter $m$ for $\tau=0.1$ (curve 1), 0.5 (curve 2), 1 (curve 3). 
where $\varphi$ and $\chi$ are linear perturbation terms. After substituting (10) into (1), it is straightforward to derive

$$
\left(\begin{array}{c}
\partial \varphi / \partial t \\
\partial \chi / \partial t
\end{array}\right)=\hat{L}\left(\begin{array}{l}
\varphi \\
\chi
\end{array}\right)
$$

where

$$
\hat{L}=\left(\begin{array}{cc}
\partial_{\xi}^{2}+m V U^{m-1}+c \partial_{\xi} & U^{m} \\
-m V U^{m-1} & \tau \partial_{\xi}^{2}-U^{m}+c \partial_{\xi}
\end{array}\right)
$$

The stability of the travelling front is then defined from the spectra of $\hat{L}$. It is straightforward to show that the essential spectra of this operator always lies in the left half plane and therefore the discrete spectra is solely responsible for the transition to instability. We seek the solution of (11) of the form

$$
\varphi(\xi, t)=\varphi(\xi) e^{\lambda t}, \quad \chi(\xi, t)=\chi(\xi) e^{\lambda t},
$$

where $\lambda$ is a spectral parameter. If the operator $\hat{L}$ has points of discrete spectra with $\Re(\lambda)>0$ then the travelling front solution of (1) is unstable. Substituting (13) into (11) and introducing a vector with the components $z_{1}=\varphi, z_{2}=\varphi_{\xi}, z_{3}=\chi, z_{4}=\chi_{\xi}$ we obtain the system of ODEs in the form

$$
\dot{\mathbf{z}}=\mathbf{A z}
$$

where

$$
\mathbf{A}=\left(\begin{array}{cccc}
0 & 1 & 0 & 0 \\
\lambda-m V U^{m-1} & -c & -U^{m} & 0 \\
0 & 0 & 0 & 1 \\
\frac{m}{\tau} V U^{m-1} & 0 & \frac{\lambda+U^{m}}{\tau} & -\frac{c}{\tau}
\end{array}\right)
$$

Using the fact that $U \rightarrow 1, V \rightarrow 0$ as $\xi \rightarrow-\infty$ and $U \rightarrow 0$, $V \rightarrow 1$ as $\xi \rightarrow+\infty$ it is easy to obtain the limit matrices $\mathbf{A}^{ \pm}(\lambda)=$ 
$\lim _{\xi \rightarrow \pm \infty} \mathbf{A}(\xi, \lambda)$. The eigenvalues of $\mathbf{A}^{+}$and $\mathbf{A}^{-}$are

$$
\begin{array}{ll}
\mu_{1,2}^{-}(\lambda)=\frac{-c \mp \sqrt{c^{2}+4 \lambda}}{2}, & \mu_{1,2}^{+}=\mu_{1,2}^{-}, \\
\mu_{3,4}^{-}(\lambda)=\frac{-c \mp \sqrt{c^{2}+4 \tau(\lambda+1)}}{2 \tau}, & \mu_{3,4}^{+}(\lambda)=\mu_{3,4}^{-}(\lambda-1),
\end{array}
$$

with corresponding eigenvectors denoted by $\zeta_{i}^{ \pm}$which will be considered as normalized. The limit matrix $\mathbf{A}^{-}$has two eigenvalues $\mu_{2}^{-}$ and $\mu_{4}^{-}$with positive real parts. Let us represent the 2-dimensional space of solutions of equation (14) bounded at $\xi=-\infty$ as

$$
Z^{-}=\sum_{i=2,4} \alpha_{i} z_{i}^{-}(\xi, \lambda)
$$

where $\alpha_{i}$ are constant coefficients and $z_{i}^{-}(\xi, \lambda)=\left(z_{i 1}^{-}, z_{i 2}^{-}, z_{i 3}^{-}, z_{i 4}^{-}\right)^{T}$ are the solutions of (14) satisfying

$$
\lim _{\xi \rightarrow \infty} \exp \left(-\mu_{i}^{-} \xi\right) z_{i}^{-}(\xi, \lambda)=\zeta_{i}^{-}, \quad i=2,4 .
$$

Similarly limit matrix $\mathbf{A}^{+}$has two eigenvalues $\mu_{1}^{+}$and $\mu_{3}^{+}$with negative real part and we introduce a space of solutions bounded at $\xi=+\infty$

$$
Z^{+}=\sum_{i=1,3} \beta_{i} z_{i}^{+}(\xi, \lambda),
$$

where $\beta_{i}$ are constants and

$$
\lim _{\xi \rightarrow \infty} \exp \left(-\mu_{i}^{+} \xi\right) z_{i}^{+}(\xi, \lambda)=\zeta_{i}^{+}, \quad i=1,3 .
$$

If $\lambda$ is a point of discrete spectra then equation (14) has a solution bounded both at $\xi= \pm \infty$ and we require the following matching conditions at $\xi=0$ to be satisfied

$$
\sum_{i=2,4} \alpha_{i} z_{i}^{-}(0, \lambda)=\sum_{i=1,3} \beta_{i} z_{i}^{+}(0, \lambda) .
$$


The last equation is algebraic for the four coefficients $\alpha_{2,4}$ and $\beta_{1,3}$ if $z_{2,4}^{-}(0, \lambda)$ and $z_{1,3}^{+}(0, \lambda)$ are known. It has a nontrivial solution if

$$
D(\lambda)=0,
$$

where

$$
D(\lambda)=\left|\begin{array}{llll}
z_{21}^{-}(0, \lambda) & z_{41}^{-}(0, \lambda) & z_{11}^{+}(0, \lambda) & z_{31}^{+}(0, \lambda) \\
z_{22}^{-}(0, \lambda) & z_{42}^{-}(0, \lambda) & z_{12}^{+}(0, \lambda) & z_{32}^{+}(0, \lambda) \\
z_{23}^{-}(0, \lambda) & z_{43}^{-}(0, \lambda) & z_{13}^{+}(0, \lambda) & z_{33}^{+}(0, \lambda) \\
z_{24}^{-}(0, \lambda) & z_{44}^{-}(0, \lambda) & z_{14}^{+}(0, \lambda) & z_{34}^{+}(0, \lambda)
\end{array}\right|
$$

We will refer to $D(\lambda)$ as the Evans function. Similar definitions of the Evans function are given in $[7,9]$. Now according to the method introduced in [9] we should take one of the vectors $\zeta_{2,4}^{-}$as the initial conditions at $\xi=-\ell_{1}$ and integrate (14) until $\xi=\ell_{2}$, where $\ell_{1,2}$ are sufficiently large. A special technique of integration is introduced to avoid exponential divergence of the solution. Then we represent the solution at $\xi=\ell_{2}$ as a superposition of two solutions $z_{2,4}$ unbounded as $\xi \rightarrow+\infty$ and satisfying $\lim _{\xi \rightarrow \infty} \exp \left(-\mu_{i}^{+} x\right) z_{i}=\zeta_{i}^{+}$, $i=2,4$. This procedure gives us four coefficients (two for each value of initial conditions $\zeta_{2}^{-}$or $\zeta_{4}^{-}$) which are sometimes called transmission coefficients. A determinant can then be constructed using these coefficients and the Evans function can be defined then via this determinant(see [7] for details). A more precise definition based on the solutions of the problem adjoint to (14) can be found in $[10]$.

However, the method of the Evans function calculation briefly outlined above has difficulties for the problem presented here. The main difficulty is that it can be applied only to a class of rather specific systems which have one dimensional space of solutions bounded (or unbounded) at both $\xi=+\infty$ or $-\infty$. This is not the case in our problem where we have the example of a stiff system (see [12] for definition). There are two coefficients $\mu_{2,4}^{ \pm}$, one is always bigger 
than the other (except in some special case when they are equal for some value of $\lambda$ ). We are not able to trace the solution which corresponds to the lower coefficient of exponential growth numerically, because another solution is always excited due to the errors of numerical integration if we use a standard integration method like the fourth order Runge-Kutta, which is not appropriate to treat the stiff systems. This is the reason why we can manage to calculate only one transmission coefficient between solutions with maximal exponential growth at $\xi= \pm \infty$. This, however, is not sufficient for investigating the stability of the travelling front.

A way to overcome this difficulty was proposed in $[12,13]$. The authors introduced an alternative definition of the Evans function which numerically implies using the compound matrix method [14, $15,16]$. We shall now proceed to describe this method.

\section{Compound matrix method}

Let $z_{2}$ and $z_{4}$ be two solutions of (14) bounded at $\xi=-\infty$. Consider the $4 \times 2$ solution matrix

$$
\boldsymbol{\Phi}(\xi)=\left(\begin{array}{llll}
z_{21} & z_{22} & z_{23} & z_{24} \\
z_{41} & z_{42} & z_{43} & z_{44}
\end{array}\right)^{T} .
$$

We arrange the $2 \times 2$ minors of $\boldsymbol{\Phi}$ to a vector with the components

$$
\begin{array}{ll}
\phi_{1}=z_{21} z_{42}-z_{41} z_{22}, & \phi_{4}=z_{22} z_{43}-z_{42} z_{23}, \\
\phi_{2}=z_{21} z_{43}-z_{41} z_{23}, & \phi_{5}=z_{22} z_{44}-z_{42} z_{24}, \\
\phi_{3}=z_{21} z_{44}-z_{41} z_{24}, & \phi_{6}=z_{23} z_{44}-z_{43} z_{24} .
\end{array}
$$

We call $\phi$ the second compound of $\boldsymbol{\Phi}$ and it satisfies the equation

$$
\dot{\phi}=\mathbf{B} \phi,
$$


where $\mathbf{B}$ is a $6 \times 6$ matrix those elements are found from the spectral problem for operator $L(12)$ (see $[14,15]$ for details). It can be shown that the eigenvalues $s_{i}^{ \pm}$of $\mathbf{B}$ in the limits $\xi= \pm \infty$ are

$$
\begin{array}{ll}
s_{1}^{ \pm}=\mu_{1}^{ \pm}+\mu_{2}^{ \pm}, & s_{4}^{ \pm}=\mu_{2}^{ \pm}+\mu_{3}^{ \pm}, \\
s_{2}^{ \pm}=\mu_{1}^{ \pm}+\mu_{3}^{ \pm}, & s_{5}^{ \pm}=\mu_{2}^{ \pm}+\mu_{4}^{ \pm}, \\
s_{3}^{ \pm}=\mu_{1}^{ \pm}+\mu_{4}^{ \pm}, & s_{6}^{ \pm}=\mu_{3}^{ \pm}+\mu_{4}^{ \pm} .
\end{array}
$$

See that the two-dimensional space of solutions of (14) bounded at $\xi=-\infty$ degenerates to one solution $\phi^{-}(\xi)$ of the equation (26) with the largest rate of exponential decay $s_{5}^{-}$as $\xi \rightarrow-\infty$. Similarly, the two-dimensional space of solutions of (14) bounded at $\xi=+\infty$ degenerates to the solution $\phi^{+}(x)$ of the equation (26) with the largest rate of exponential decay $s_{3}^{+}$as $\xi \rightarrow+\infty$. In this case localized solutions of (26) and corresponding values of $\lambda$ can be found using the method introduced in [9]. After numerical integration of (26) from $\xi=\ell^{-}$to $\xi=0$ with appropriate initial conditions we can find the approximation of the solutions of (14) exponentially decaying as $\xi \rightarrow-\infty$ by inversion of the system (25). In the same way integration of (26) from $\xi=\ell^{+}$to $\xi=0$ gives us the solutions of (14) exponentially decaying as $\xi \rightarrow+\infty$. Then we define the Evan's function via $4 \times 4$ determinant (23). However it is much easier to express this determinant in terms of $\phi^{ \pm}$

$$
D(\lambda)=\left(\phi^{-}(0) \cdot \Sigma \phi^{+}(0)\right),
$$

where $(\cdot)$ denotes scalar product of two vectors and

$$
\Sigma=\left(\begin{array}{cccccc}
0 & 0 & 0 & 0 & 0 & 1 \\
0 & 0 & 0 & 0 & -1 & 0 \\
0 & 0 & 0 & 1 & 0 & 0 \\
0 & 0 & 1 & 0 & 0 & 0 \\
0 & -1 & 0 & 0 & 0 & 0 \\
1 & 0 & 0 & 0 & 0 & 0
\end{array}\right)
$$


The problem of stability of the travelling front of (1) reduces to the search for zeros of the Evans function (28) located in the right half plane. Zeros of $D(\lambda)$ can be calculated using argument principle. The number of zeros in the right half plane equals to the number of times the image of the imaginary axis under $D(i t), t \in R$ winds around the origin. Pictures of $D(i t), t \in R$ are called Nyquist plots. Figure 3 shows Nyquist plots for $\tau=0.1$ and $m=8.2$, $8.35,8.6$. For $m=8.2$ the curve does not encircle the origin, hence the travelling front is stable. Transition to instability occurs for $m=8.35$, when two complex conjugate eigenvalues cross the imaginary axes and the curve passes through the origin three times. The front is clearly unstable for $m=8.6$ when the curve encircles the origin two times and therefore there are two points of discrete spectra in right half plane.

\section{Conclusions}

In this work we have investigated propagation of travelling front solutions on the example of an autocatalytic reaction involving two diffusing chemicals in one spatial dimension using two methods: shooting and relaxation. Both methods were successful in obtaining solutions.

The Evans function method was employed to examine the linear stability problem. It was shown that the compound matrix method significantly expands the applicability of the Evans function approach. Although in this work we found simple numerical criteria for the transition to instability for steady propagating solutions, we believe that an advanced Evans function method would provide more detailed information such as the location and the number of points of discrete spectra and the corresponding modes for 


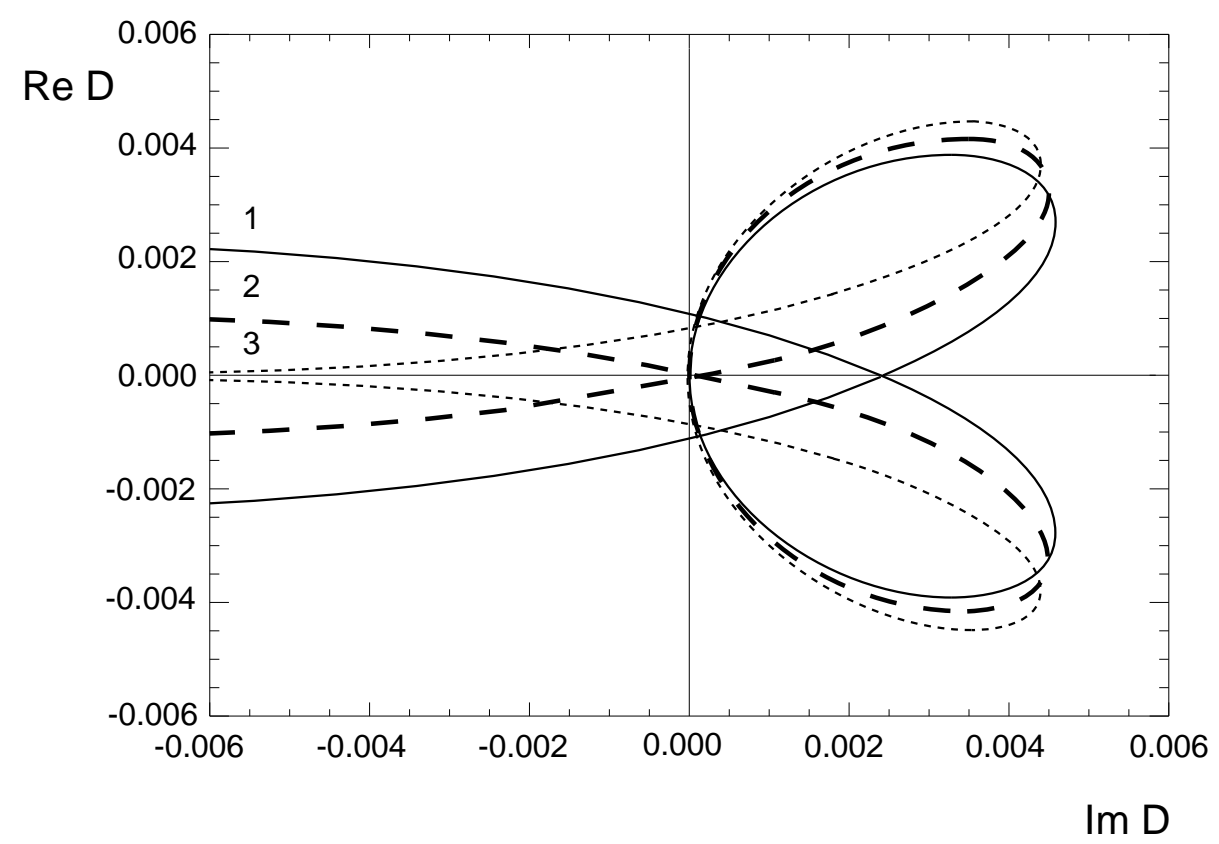

Figure 3: Transition to instability. The image of the imaginary axis $D(i t)$ near the origin for $t=0.1$ and for $m=8.2$ (curve 1 ), 8.35 (curve 2), 8.6 (curve 3). 
the linearized stability problem.

We are currently in the process of applying these methods to a similar system with Arrhenius kinetics which describes the propagation of combustion waves.

\section{References}

[1] A. I. Volpert, V. A. Volpert, and V. A. Volpert, Traveling Wave Solutions of Parabolic Systems, Trans. Math.

Monographs vol. 140. C272

[2] J. D. Murray. Lectures on Nonlinear-Differential-Equation Models in Biology. Claredon Press, Oxford, 1977. C272

[3] Ya. B. Zeldovich, G. I. Barenblatt, V. B. Librovich, and G. M. Makhviladze. Mathematical Theory of Combustion and Explosions. Consultants Bureau, New York and London, 1985. $\mathrm{C} 272$

[4] G. P. Agrawal. Nonlinear Fiber Optics. Academic Press, New York, 1988. C272

[5] B. Sandstede. Stability of travelling waves. In "Handbook of Dynamical Systems II" (B. Fiedler, ed.). Elsevier, (2002) 983-1055. C272

[6] W. H. Press, S. A. Teukolsky, W. T. Vetterling, and B. P. Flannery. Numerical Recipes in C: The Art of Scientific Computing. Cambridge University Press, 1992. C273, C275, C276 
[7] N. J. Balmforth, R. V. Craster, and S. J. A. Malham, Unsteady fronts in an autocatalytic systems, Proc. $R$. Soc. Lond. A, 455 (1999), 1401-1433. C273, C274, C282

[8] R. O. Weber, G. N. Mercer, H. S. Sidhu and B. F. Gray, Combustion waves for gases $(L e=1)$ and solids $(L e \rightarrow \infty)$, Proc. R. Soc. Lond. A, 453 (1997), 1105-1118. C273

[9] R. L. Pego, P. Smereka, and M. I. Weinstein, Oscillatory instability of traveling waves for a $\mathrm{KdV}$-Burgers equation, Physica D, 67 (1993), 45-65. C273, C282, C284

[10] J. Swinton and J. Elgin, Stability of travelling pulse solutions to a laser equation, Phys. Lett. A, 145 (1990), 428-433. C282

[11] T. J. Bridges and G. Derks, The symplectic Evans matrix, and the instability of solitary waves and fronts with symmetry, Arch. Rat. Mech. Anal. 156(2001), pp. 1-87. C273

[12] A. L. Afendikov and T. J. Bridges, Instability of the Hocking-Stewartson pulse and its implications for three-dimensional Poiseuille flow, Proc. R. Soc. Lond. A, 457 (2001), pp. 1-16. C273, C282, C283

[13] L. A. Allen and T. J. Bridges, Numerical exterior algebra and the compound matrix method, Numerische Mathematik 92 (2002), 197-232. C273, C283

[14] B. S. Ng and W. H. Reid, An initial value Method for eigenvalue problems using compound matrices, J. Comput. Phys., 30 (1979), 125-136. C273, C283, C284

[15] B. S. Ng and W. H. Reid, The compound matrix method for ordinary differential equation, J. Comput. Phys., 58 (1985), 209-228. C273, C283, C284 
[16] P. G. Drazin and W. H. Reid, Hydrodynamic stability. Cambridge University Press, 1981. C273, C283

[17] J. R. Cash and A. Singhal, High order methods for the numerical solution of two-point boundary value problems, BIT 22 (1982), 184-199. C276 